\title{
Multiple nodules in the scrotal wall
}

\author{
kouki chaima ${ }^{1}$, mariem Amouri ${ }^{1}$, and Hamida Turki ${ }^{1}$ \\ ${ }^{1}$ Hedi Chaker Hospital
}

October 20, 2021

Title: Multiple nodules in the scrotal wall

Presentation:

A 52-year-old male with no significant past medical history presented to our department with multiple scrotal nodules. The lesions had gradually increased in number and size during the last 2 decades without causing any discomfort aside cosmetic concern. Physical examination (Fig. 1) revealed prominent 2 to 20 $\mathrm{mm}$ yellowish or white firm papules or nodules on the scrotum. There were no areas of ulceration, discharge or infection. Laboratory examination including serum calcium, phosphorus, thyroid hormone and alkaline phosphatase levels showed no abnormality.

What's your diagnosis?

Histological examination of an excised nodule reveals extensive intradermal deposition of calcium surrounded by histiocytes without any cystic structure. The diagnosis of idiopathic scrotal calcinosis (ISC) was retained and the patient was referred to the surgical department for wide excision.

Scrotal nodules and cysts are uncommon. Since they are often asymptomatic, the diagnostic may be delayed for several years or decades. The aesthetic discomfort represents the main complaint and may severely affect sexual life. Herein, we repot a rare case of extensive ISC. It affects dark colored skin men mainly aged from 20 to 40 years old, suggesting an ethnic susceptibility. ${ }^{1}$ Despite many theories that have been developed over the years including calcification of epidermoid cysts secondary to infections, eccrine epithelial cysts or degenerated dartos muscle, pathogenesis of scrotal calcinosis remains controversial. It arises either on preexisting cysts like sebaceous cysts or steatocystoma multiplex or develops de novo. The latter is designated as idiopathic scrotal calcinosis (ISC). The major difference from calcified cysts is the complete absence of a lining epithelium. ${ }^{2}$

There is no association with calcium metabolic diseases or other systemic comorbidities. ${ }^{1}$ Surgery is the mainstay of the treatment providing excellent results. ${ }^{2}$

\section{References:}

1-Ahlam A, Hassam B. Scrotal calcinoses [in French] Pan Afr Med J. 2014;18:297.

2-Killedar MM, Shivani AA, Shinde U. Idiopathic scrotal calcinosis. IndianJ Surg 2016; 78: 329- 30.

\section{Legend figure :}

Fig1: Multiple yellowish firm nodules on the scrotal wall 


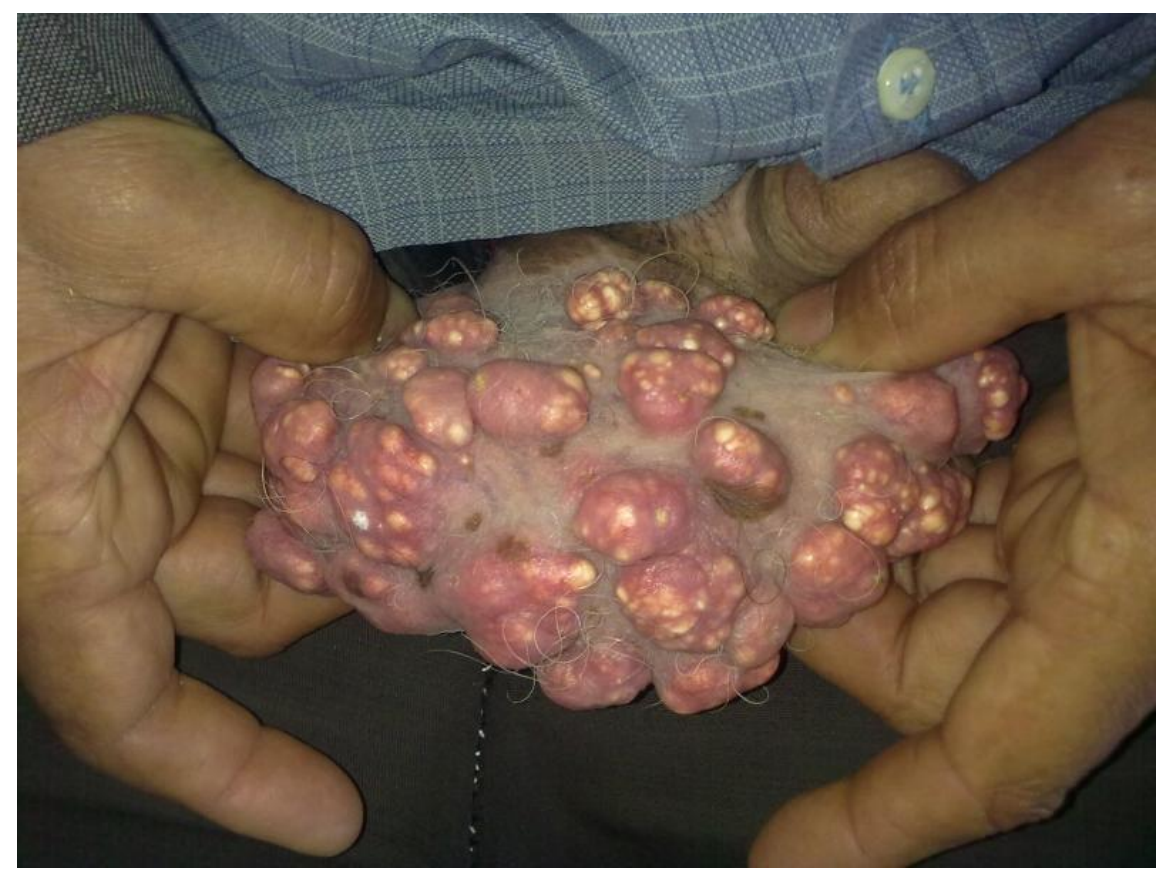

